\title{
Independence of higher Kurepa hypotheses
}

\author{
Sy-David Friedman • Mohammad Golshani
}

Received: 30 August 2011 / Accepted: 23 April 2012 / Published online: 13 May 2012

(C) Springer-Verlag 2012

\begin{abstract}
We study the Generalized Kurepa hypothesis introduced by Chang. We show that relative to the existence of an inaccessible cardinal the Gap- $n$-Kurepa hypothesis does not follow from the Gap- $m$-Kurepa hypothesis for $m$ different from $n$. The use of an inaccessible is necessary for this result.
\end{abstract}

\section{Introduction}

In this paper we study the Generalized Kurepa hypothesis introduced by Chang (see Chapter VII of [1]). We show that relative to the existence of an inaccessible cardinal the Gap- $n$-Kurepa hypothesis does not follow from the Gap- $m$-Kurepa hypothesis for $m$ different from $n$. The use of an inaccessible is necessary for this result.

Definition 1.1 (a) For infinite cardinals $\lambda<\kappa$, a $\mathrm{KH}(\kappa, \lambda)-$ family is a family $\mathcal{F}$ of subsets of $\kappa$ such that:

(i) $\operatorname{Card}(\mathcal{F}) \geq \kappa^{+}$,

(ii) for all $x \in[\kappa]^{\lambda}, \operatorname{Card}(\mathcal{F}\lceil x) \leq \lambda$, where $\mathcal{F} \uparrow x=\{t \cap x: t \in \mathcal{F}\}$. We say $\mathrm{KH}(\kappa, \lambda)$ holds if such a family exists.

\section{S.-D. Friedman}

Kurt Godel Research Center, University of Vienna, Vienna, Austria

e-mail: sdf@logic.univie.ac.at

M. Golshani (凶)

Department of Mathematics, Shahid Bahonar University of Kerman, Kerman, Iran e-mail: golshani.m@gmail.com 
(b) For infinite cardinals $\lambda \leq \kappa$, a $\mathrm{KH}(\kappa,<\lambda)$-family is a family $\mathcal{F}$ of subsets of $\kappa$ such that:

(i) $\operatorname{Card}(\mathcal{F}) \geq \kappa^{+}$,

(ii) for all $x \in[\kappa]^{<\lambda}, \operatorname{Card}\left(\mathcal{F}\lceil x) \leq \operatorname{Card}(x)+\aleph_{0}\right.$.

We say $\mathrm{KH}(\kappa,<\lambda)$ holds if such a family exists.

(c) Let $n \geq 1, n$ finite. By the Gap- $n$-Kurepa hypothesis we mean the following statement: for all infinite cardinals $\lambda, \operatorname{KH}\left(\lambda^{+n}, \lambda\right)$ holds.

The following is well-known (see [1], Chapter VII, Theorems 3.2 and 3.3).

Theorem 1.2 (Jensen). If $V=L$, then $\mathrm{KH}\left(\kappa,<\lambda^{+}\right.$) (and hence $\mathrm{KH}(\kappa, \lambda)$ ) holds for all infinite cardinals $\lambda<\kappa, \kappa$ regular.

In this paper we prove the following theorem.

Theorem 1.3 Let $n \geq 1$. The following are equiconsistent:

(a) There exists an inaccessible cardinal,

(b) $\mathrm{GCH}+$ the Gap-m-Kurepa hypothesis holds for all $m \neq n$, but the Gap-n-Kurepa hypothesis fails.

Remark 1.4 Our proof shows that if $\lambda<\kappa$ are infinite cardinals, $\kappa$ regular and $\mathrm{KH}(\kappa, \lambda)$ fails, then $\kappa^{+}$is inaccessible in $L$ (see Lemma 3.1).

Remark $1.5(b)$ of the above Theorem can be strengthened to the Gap- $m$-Kurepa hypothesis holds for all $m \neq n$, but $\mathrm{KH}\left(\aleph_{n}, \aleph_{0}\right)$ fails (see Lemma 2.7).

\section{Proof of $\operatorname{Con}(a)$ implies $\operatorname{Con}(b)$}

In this section we show that if there exists an inaccessible cardinal, then in a forcing extension of $L$, the Gap- $m$-Kurepa hypothesis holds for all $m \neq n$, but the Gap- $n$ Kurepa hypothesis fails, where $n \geq 1$ is a fixed natural number.

From now on assume that $V=L$, and let $\kappa$ be an inaccessible cardinal. We consider two cases.

Case 1. $n=1$.

Let $\mathbb{P}=\operatorname{Col}\left(\omega_{1},<\kappa\right)$ be the Levy collapse with countable conditions which converts $\kappa$ into $\omega_{2}$, and let $G$ be $\mathbb{P}$-generic over $L$.

Lemma 2.1 The following hold in $L[G]$ :

(a) $\mathrm{KH}\left(\aleph_{1}, \aleph_{0}\right)$ fails,

(b) The Gap-m-Kurepa hypothesis holds for all $m \geq 2$.

Proof (a) is a well known result of Silver (see [7], or [2] Lemma 20.4).

(b) Let $m \geq 2$, and let $\lambda$ be an infinite cardinal in $L[G]$. Let $\mu=\left(\lambda^{+m}\right)^{L[G]}$. By Theorem 1.2 , there is a $\mathrm{KH}(\mu, \lambda)$ family $\mathcal{F}$ in $L$. We show that it remains a $\operatorname{KH}(\mu, \lambda)$ family in $L[G]$. Clearly $\operatorname{Card}(\mathcal{F})=\mu^{+L}=\left(\lambda^{+m+1}\right)^{L[G]}$. Suppose $x \in\left([\mu]^{\lambda}\right)^{L[G]}$. 
Note that $\mathbb{P}$ is $\kappa-c . c$. and $\omega_{1}-$ closed, and in $L[G], \kappa$ becomes $\omega_{2}$. Thus it is easily seen that infinite sets in $L[G]$ are covered by sets of the same cardinality which belong to the ground model $L$, in particular there is a set $y \subseteq \mu$ in $L$ such that $x \subseteq y$ and $x$ and $y$ have the same cardinality in $L[G]$. If $\lambda \neq \aleph_{1}$, then $y$ has $L-$ cardinality $\lambda$, hence in $L, \operatorname{Card}(\mathcal{F} \backslash y) \leq \lambda$. It follows that in $L[G], \operatorname{Card}(\mathcal{F} \backslash x) \leq \operatorname{Card}(\mathcal{F} \backslash y) \leq \lambda$. If $\lambda=\aleph_{1}$, then $y$ has $L$-cardinality less than $\kappa$, hence in $L, \operatorname{Card}(\mathcal{F}\lceil y)<\kappa$. It follows that in $L[G], \operatorname{Card}\left(\mathcal{F}\lceil y) \leq \aleph_{1}\right.$, and hence in $L[G], \operatorname{Card}(\mathcal{F}\lceil x) \leq \operatorname{Card}(\mathcal{F}\lceil y) \leq$ $\aleph_{1}=\lambda$.

Case 2. $n \geq 2$.

For each $i, 0<i<n$, fix an injection $J_{i}:\left[\omega_{n}\right]^{\leq \omega_{i}} \longrightarrow \omega_{n}$. Let $\mathbb{R}=\mathbb{P} \times$ $\prod_{0<i<n} \mathbb{Q}_{i}$, where the forcing notions $\mathbb{P}$ and $\mathbb{Q}_{i}, 0<i<n$, are defined as follows. $\mathbb{P}=\operatorname{Col}\left(\omega_{n},<\kappa\right)$ is the Levy collapse with conditions of size $<\omega_{n}$ which converts $\kappa$ into $\omega_{n+1}$.

$\mathbb{Q}_{i}, 0<i<n$, is the set of triples $p=\left(X_{p}, \mathcal{F}_{p}, g_{p}\right)$ such that:

$(i-1) X_{p}$ is a subset of $\omega_{n}$ of size $\leq \omega_{i}$,

$(i-2) \mathcal{F}_{p}$ is a subset of $X_{p} 2$ of size $\leq \omega_{i}$,

$(i-3) g_{p}$ is a $1-1$ function from a subset of $\kappa$ into $\mathcal{F}_{p}$,

$(i-4) \mathcal{F}_{p}$ is $\omega_{i}$-closed in the following sense: If $t \in X_{p} 2$ and $\left\langle X_{\xi}: \xi<\omega_{i-1}\right\rangle$ is a sequence of subsets of $X_{p}$ such that for all $\xi<\omega_{i-1}, J_{i}\left(X_{\xi}\right) \in X_{p}$ and $t \uparrow X_{\xi} \in \mathcal{F}_{p} \uparrow$ $X_{\xi}$, then there is $s \in \mathcal{F}_{p}$ such that $s \uparrow X=t \uparrow X$ and $s \uparrow\left(X_{p} \backslash X\right)=0 \uparrow\left(X_{p} \backslash X\right)$ (=the zero function on $X_{p} \backslash X$ ), where $X=\bigcup_{\xi<\omega_{i-1}} X_{\xi}$.

For $p, q \in \mathbb{Q}_{i}$, let $p \leq q$ ( $p$ is an extension of $q$ ) iff:

$(i-5) X_{p} \supseteq X_{q}$,

$(i-6) \mathcal{F}_{q}=\mathcal{F}_{p} \uparrow X_{q}$,

$(i-7) \operatorname{dom}\left(g_{p}\right) \supseteq \operatorname{dom}\left(g_{q}\right)$,

$(i-8)$ for all $\alpha \in \operatorname{dom}\left(g_{q}\right), g_{q}(\alpha)=g_{p}(\alpha) \uparrow X_{q}$.

We show that in the generic extension by $\mathbb{R}$, the Gap- $m$-Kurepa hypothesis holds for all $m \neq n$, but the Gap- $n$-Kurepa hypothesis fails.

Lemma 2.2 (a) $\mathbb{P}$ is $\omega_{n}$-closed,

(b) $\mathbb{P}$ satisfies the $\kappa$-c.c.,

(c) Let $0<i<n$. Then $\mathbb{Q}_{i}$ is $\omega_{i+1}$-closed modulo $J_{i}$ in the following sense: If $\left\langle p_{\xi}: \xi<\lambda\right\rangle, \lambda \leq \omega_{i}$, is a descending sequence of conditions in $\mathbb{Q}_{i}$ such that for all $\xi<\lambda, J_{i}\left(X_{p_{\xi}}\right) \in X_{p_{\xi+1}}$, then there is a condition $p \in \mathbb{Q}_{i}$ which extends all of the $p_{\xi}$ 's, $\xi<\lambda$. Furthermore if $\lambda<\omega_{i}$, then $p$ can be chosen to be the greatest lower bound of the $p_{\xi}$ 's, $\xi<\lambda$.

(d) Let $0<i<n$. Then $\mathbb{Q}_{i}$ has the $\omega_{i+2}-$ c.c.

Proof $(a)$ and (b) are well known results of Levy (see [2], Lemma 20.4). We prove (c) and $(d)$.

(c) Fix $0<i<n$, and let $\left\langle p_{\xi}: \xi<\lambda\right\rangle$ be as above. To simplify the notation let $p_{\xi}=\left(X_{\xi}, \mathcal{F}_{\xi}, g_{\xi}\right), \xi<\lambda$. We consider two cases.

Case 1. $\lambda<\omega_{i}$.

Let $p=(X, \mathcal{F}, g)$, where: 
- $X=\bigcup_{\xi<\lambda} X_{\xi}$,

- $\mathcal{F}$ is the least subset of ${ }^{X} 2$ such that if $t \in \in^{X} 2$ and for all $\xi<\lambda, t\left\lceil X_{\xi} \in \mathcal{F}_{\xi}\right.$ then $t \in \mathcal{F}$, and $\mathcal{F}$ is $\omega_{i}$-closed in the sense of $(i-4)$,

- $\operatorname{dom}(g)=\bigcup_{\xi<\lambda} \operatorname{dom}\left(g_{\xi}\right)$,

- for all $\alpha \in \operatorname{dom}(g), g(\alpha)=\bigcup\left\{g_{\xi}(\alpha): \xi<\lambda, \alpha \in \operatorname{dom}\left(g_{\xi}\right)\right\}$.

It is easy to show that $p \in \mathbb{Q}_{i}$ and that $p$ is the greatest lower bound for the sequence $\left\langle p_{\xi}: \xi<\lambda\right\rangle$.

Case 2. $\lambda=\omega_{i}$.

Let $p=(X, \mathcal{F}, g)$, where:

- $X=\bigcup_{\xi<\lambda} X_{\xi}$,

- $\operatorname{dom}(g)=\bigcup_{\xi<\lambda} \operatorname{dom}\left(g_{\xi}\right)$,

- for all $\alpha \in \operatorname{dom}(g), g(\alpha)=\bigcup\left\{g_{\xi}(\alpha): \xi<\lambda, \alpha \in \operatorname{dom}\left(g_{\xi}\right)\right\}$,

- $\mathcal{F}$ is the least subset of ${ }^{X} 2$ such that $\operatorname{ran}(g) \cup\left\{t \mid X_{\xi} \cup 0\left\lceil\left(X \backslash X_{\xi}\right): t \in X_{\xi}\right\} \subseteq \mathcal{F}\right.$ and $\mathcal{F}$ is $\omega_{i}$-closed in the sense of $(i-4)$.

Then it is easy to show that $p \in \mathbb{Q}_{i}$ and that $p$ is a lower bound for the sequence $\left\langle p_{\xi}: \xi<\lambda\right\rangle$.

(d) Fix $0<i<n$. Suppose that $\mathbb{Q}_{i}$ does not satisfy the $\omega_{i+2}-$ c.c. Let $A$ be a maximal antichain in $\mathbb{Q}_{i}$ of size $\geq \omega_{i+2}$. By a $\Delta$-system argument we can assume that

- The sequence $\left\langle X_{p}: p \in A\right\rangle$ forms a $\Delta$-system with root $X$.

- The sequence $\left\langle\operatorname{dom}\left(g_{p}\right): p \in A\right\rangle$ forms a $\Delta$-system with root $D$.

- For all $p \neq q$ in $A, g_{p}\left\lceil D=g_{q}\left\lceil D\right.\right.$ and $\mathcal{F}_{p}\left\lceil X=\mathcal{F}_{q}\lceil X\right.$.

Let $\theta$ be large regular, and let $M$ be an elementary submodel of $H(\theta)$ of size $\omega_{i+1}$ which is closed under $\omega_{i}-$ sequences and such that $\mathbb{Q}_{i}, X, D, A \in M$. Pick $q \in A \backslash M$ and let $q\left\lceil M=\left(X_{q}\left\lceil M, \mathcal{F}_{q}\left\lceil M, g_{q}\lceil M)\right.\right.\right.\right.$, where:

- $X_{q}\left\lceil M=X_{q} \cap M\right.$,

- $\mathcal{F}_{q}\left\lceil M=\left\{t \uparrow\left(X_{q} \cap M\right): t \in F_{q}\right\}\right.$,

- $\operatorname{dom}\left(g_{q}\lceil M)=\operatorname{dom}\left(g_{q}\right) \cap M\right.$,

- for all $\alpha \in \operatorname{dom}\left(g_{q}\lceil M),\left(g_{q}\lceil M)(\alpha)=g_{q}(\alpha)\left\lceil\left(X_{q} \cap M\right)\right.\right.\right.$.

Then $q\left\lceil M \in \mathbb{Q}_{i} \cap M\right.$. Extend this condition to a condition $p \in \mathbb{Q}_{i} \cap M$ which extends an element $r \in A$. We show that $p$ and $q$ and hence $r$ and $q$ are compatible, which is impossible since $r, q \in A$.

Fix $s_{0} \in \mathcal{F}_{p}, t_{0} \in \mathcal{F}_{q}$. Define $X, \mathcal{F}$ and $g$ as follows:

- $X=X_{p} \cup X_{q}$,

- $\mathcal{F}$ is the least subset of ${ }^{X} 2$ such that $\left\{s\left\lceil X_{p} \cup t\left\lceil\left(X_{q} \backslash M\right): s \in \mathcal{F}_{p}, t \in \mathcal{F}_{q}\right\} \subseteq \mathcal{F}\right.\right.$, and $\mathcal{F}$ is $\omega_{i}$-closed in the sense of $(i-4)$,

- $\operatorname{dom}(g)=\operatorname{dom}\left(g_{p}\right) \cup \operatorname{dom}\left(g_{q}\right)$,

- $g(\alpha)= \begin{cases}g_{p}(\alpha) \uparrow X_{P} \cup g_{q}(\alpha) \uparrow\left(X_{q} \backslash M\right) & \text { if } \alpha \in \text { domg }_{q} \cap M, \\ g_{p}(\alpha) \uparrow X_{P} \cup t_{0} \uparrow\left(X_{q} \backslash M\right) & \text { if } \alpha \in \text { domg }_{p} \backslash \text { domg } \\ & \text { if }, \\ g_{q} \uparrow X_{q} \cup s_{0} \uparrow\left(X_{p} \backslash X_{q}\right) & \text { if } \alpha \in \text { domg }_{q} \backslash M .\end{cases}$ 
Then $(X, \mathcal{F}, g) \in \mathbb{Q}_{i}$ and it extends both of $p$ and $q$.

Let $K=G \times \prod_{0<i<n} H_{i}$ be $\mathbb{R}=\mathbb{P} \times \prod_{0<i<n} \mathbb{Q}_{i}$ generic over $L$. It follows from the above lemma that

- $\omega_{i}^{L[K]}=\omega_{i}^{L}$ for all $i \leq n$.

- $\omega_{n+1}^{L[K]}=\kappa^{L}$.

Lemma 2.3 In $L[K]$, the Gap-m-Kurepa hypothesis holds for all $m \neq n$.

Proof First show that $\mathrm{KH}\left(\aleph_{n}, \aleph_{i}\right)$ holds in $L[K]$, for all $0<i<n$.

Claim 2.4 Let $0<i<n$. Forcing with $\mathbb{Q}_{i}$ adds a family $\mathcal{F} \subseteq \omega_{n} 2$ such that

(a) $\operatorname{Card}(\mathcal{F})=\kappa$,

(b) for all $X \in\left(\left[\omega_{n}\right]^{\omega_{i}}\right)^{L}, \operatorname{Card}(\mathcal{F} \uparrow X) \leq \aleph_{i}$.

Proof By Lemma 2.2, $\mathbb{Q}_{i}$ is a cardinal preserving forcing notion. It is easy to prove the following (where $H_{i}$ is assumed to be a $\mathbb{Q}_{i}$-generic filter over $L$ ):

- $\bigcup\left\{X_{p}: p \in H_{i}\right\}=\omega_{n}$,

- $\left.\bigcup \operatorname{dom}\left(g_{p}\right): p \in H_{i}\right\}=\kappa$,

- for all $X \in\left(\left[\omega_{n}\right]^{\omega_{i}}\right)^{L}$, there is some $p \in H_{i}$ with $X_{q} \supseteq X$,

- if $\alpha<\kappa$, then $g(\alpha): \omega_{n} \longrightarrow 2$, where

$$
g(\alpha)=\bigcup\left\{g_{p}(\alpha): p \in H_{i}, \alpha \in \operatorname{dom}\left(g_{p}\right)\right\}
$$

- if $\alpha<\beta<\kappa$, then $g(\alpha) \neq g(\beta)$.

Then $\mathcal{F}=\{g(\alpha): \alpha<\kappa\}$ is as required.

Claim 2.5 Infinite sets in $L[K]$ are covered by sets of the same cardinality which belong to the ground model $L$.

Proof It is easily seen that any infinite set of ordinals from $L[K]$ is covered by a set of ordinals of $L[G]$ of the same cardinality and that $L[K]$ and $L[G]$ have the same cardinals. On the other hand since $\mathbb{P}$ is $\kappa-c . c$. and $\omega_{n}-$ closed and in $L[G], \kappa$ becomes $\omega_{n+1}$, any infinite set of ordinals from $L[G]$ is covered by a set of ordinals of $L$ of the same $L[G]-$ cardinality. The result follows immediately.

Now using the above Claim and the fact that $\omega_{i}^{L[K]}=\omega_{i}^{L}$, we can show that $\mathcal{F}$ is in fact a $\mathrm{KH}\left(\aleph_{n}, \aleph_{i}\right)$ - family in $L[K]$.

Next let $\lambda$ be an infinite cardinal, $m \neq n$, and suppose $\mu=\left(\lambda^{+m}\right)^{L[K]}, \mu \neq \aleph_{n}$. We show that $\operatorname{KH}(\mu, \lambda)$ holds in $L[K]$.

Claim 2.6 $\mathrm{KH}(\mu, \lambda)$ holds in $L[G]$.

Proof If $\mu<\aleph_{n}$, the claim follows from the facts that $\mathrm{KH}(\mu, \lambda)$ holds in $L,\left(\mu^{+}\right)^{L}=$ $\left(\mu^{+}\right)^{L[G]}$ and $L$ and $L[G]$ have the same $\mu$-sequences. If $\mu>\aleph_{n}$, the claim follows exactly as in the proof of Lemma $2.1(b)$. 
Using the facts that $L[G]$ and $L[K]$ have the same cardinals and any infinite set of ordinals from $L[K]$ is covered by a set of ordinals of $L[G]$ of the same cardinality, we can immediately conclude that $\mathrm{KH}(\mu, \lambda)$ holds in $L[K]$. The Lemma follows.

Lemma 2.7 $\mathrm{KH}\left(\aleph_{n}, \aleph_{0}\right)$ fails in $L[K]$.

Before going into the details of the proof of Lemma 2.7, we introduce some notions. Let $\lambda$ be a regular cardinal, $\aleph_{n}<\lambda<\kappa$. Define the following forcing notions

$$
\begin{aligned}
\mathbb{P}_{\lambda} & =\operatorname{Col}\left(\omega_{n},<\lambda\right), \\
\mathbb{Q}_{i, \lambda} & =\text { the set of all } p \in \mathbb{Q}_{i} \text { such that } \operatorname{dom}\left(g_{p}\right) \subseteq \lambda, \\
\mathbb{R}_{\lambda} & =\mathbb{P}_{\lambda} \times \prod_{0<i<n} \mathbb{Q}_{i, \lambda}
\end{aligned}
$$

Also let $K_{\lambda}=G_{\lambda} \times \prod_{0<i<n} H_{i, \lambda}$ be $\mathbb{R}_{\lambda}$-generic over $L$. Define $\pi_{\lambda}: \mathbb{R} \longrightarrow \mathbb{R}_{\lambda}$ by

$$
\pi_{\lambda}\left(\left\langle p,\left\langle\left(X_{i}, \mathcal{F}_{i}, g_{i}\right): 0<i<n\right\rangle\right\rangle\right)=\left\langlep \left\lceil\lambda,\left\langle\left(X_{i}, \mathcal{F}_{i}, g_{i}\lceil\lambda): 0<i<n\right\rangle\right\rangle\right.\right.
$$

Claim $2.8 \pi_{\lambda}$ is a projection, i.e.

(a) $\pi_{\lambda}\left(1_{\mathbb{R}}\right)=1_{\mathbb{R}_{\lambda}}$,

(b) $\pi_{\lambda}$ is order preserving,

(c) if $r_{0} \in \mathbb{R}_{\lambda}, r_{1} \in \mathbb{R}$ and $r_{0} \leq \pi_{\lambda}\left(r_{1}\right)$, then there is some $r \leq r_{1}$ in $\mathbb{R}$ such that $\pi_{\lambda}(r) \leq r_{0}$.

Proof $(a)$ and $(b)$ are trivial. We prove $(c)$. Let $r_{j}=\left\langle p_{j},\left\langle\left(X_{i, j}, \mathcal{F}_{i, j}, g_{i, j}\right): 0<\right.\right.$ $i<n\rangle\rangle$, for $j=0,1$. Then $r=\left\langle p,\left\langle\left(X_{i}, \mathcal{F}_{i}, g_{i}\right): 0<i<n\right\rangle\right\rangle$ is as required, where:

- $p=p_{0} \cup p_{1} \uparrow(\kappa \backslash \lambda)$,

- $X_{i}=X_{i, 0}$,

- $\mathcal{F}_{i}$ is the least subset of $X_{i} 2$ such that $\mathcal{F}_{i, o} \cup\left\{t \uparrow X_{i, 1} \cup 0 \uparrow\left(X_{i, 0} \backslash X_{i, 1}\right)\right\} \subseteq \mathcal{F}_{i}$, and $\mathcal{F}_{i}$ is $\omega_{i}$-closed in the sense of $(i-4)$,

- domg $_{i}=$ domg $_{i, 0} \cup$ domg $_{i, 1}$,

- $g_{i}(\alpha)= \begin{cases}g_{i, o}(\alpha) & \text { if } \alpha \in \text { domg }_{i, 0}, \\ g_{i, 1}(\alpha) \uparrow X_{i, 1} \cup 0 \uparrow\left(X_{i, 0} \backslash X_{i, 1}\right) & \text { if } \alpha \in \text { domg }_{i, 1} \backslash \lambda .\end{cases}$

Let

$\left(\mathbb{R}: \mathbb{R}_{\lambda}\right)=\left\{\left\langle p,\left\langle\left(X_{i}, \mathcal{F}_{i}, g_{i}\right): 0<i<n\right\rangle\right\rangle \in \mathbb{R}: \pi_{\lambda}\left(\left\langle p,\left\langle\left(X_{i}, \mathcal{F}_{i}, g_{i}\right): 0<i<n\right\rangle\right\rangle\right) \in K_{\lambda}\right\}$

It follows from Lemma $2.2(c)$ that

Claim 2.9 ( $\left.\mathbb{R}: \mathbb{R}_{\lambda}\right)$ is countably closed modulo the $J_{i}$ 's, $0<i<n$, in the following sense: if $\left\langle\left\langle p_{m},\left\langle\left(X_{i, m}, \mathcal{F}_{i, m}, g_{i, m}\right): 0<i<n\right\rangle\right\rangle: m<\omega\right\rangle$ is a descending sequence of conditions in $\left(\mathbb{R}: \mathbb{R}_{\lambda}\right)$ such that for all $0<i<n$ and $m<\omega, J_{i}\left(X_{i, m}\right) \in X_{i, m+1}$, then this sequence has a lower bound in $\left(\mathbb{R}: \mathbb{R}_{\lambda}\right)$. 
Proof For each $i, 0<i<n$, the sequence $\left\langle\left(X_{i, m}, \mathcal{F}_{i, m}, g_{i, m}\right): m<\omega\right\rangle$ is a descending sequence in $\mathbb{Q}_{i}$ modulo $J_{i}$, thus by Lemma $2.2(c)$ it has a greatest lower bound $\left(X_{i}, \mathcal{F}_{i}, g_{i}\right)$. Let $r=\left\langle\bigcup_{m<\omega} p_{m},\left\langle\left(X_{i}, \mathcal{F}_{i}, g_{i}\right): 0<i<n\right\rangle\right)$. Then $r$ is the greatest lower bound for the above sequence, and $\pi_{\lambda}(r)$ is a lower bound for the sequence $\left\langle\pi_{\lambda}\left(\left\langle p_{m},\left\langle\left(X_{i, m}, \mathcal{F}_{i, m}, g_{i, m}\right): 0<i<n\right\rangle\right\rangle\right): m<\omega\right\rangle$. Note that the projection $\pi_{\lambda}$ just restricts the domain of functions involved in the condition to $\lambda$ and thus we can easily show that:

- $\pi_{\lambda}(r)$ is in fact the greatest lower bound of the above sequence.

- If $r^{\prime}$ is compatible with all of $\left\langle p_{m},\left\langle\left(X_{i, m}, \mathcal{F}_{i, m}, g_{i, m}\right): 0<i<n\right\rangle\right\rangle, m<\omega$, then $r^{\prime}$ is compatible with $\pi_{\lambda}(r)$.

It then follows from the maximality of $K_{\lambda}$ that $\pi_{\lambda}(r) \in K_{\lambda}$, and hence $r \in\left(\mathbb{R}: \mathbb{R}_{\lambda}\right)$. Thus $r$ is as required

We are now ready to prove Lemma 2.7. Assume on the contrary that $\mathrm{KH}\left(\aleph_{n}, \aleph_{0}\right)$ holds in $L[K]$. Suppose for simplicity that $1_{\mathbb{R}} \|-\left\ulcorner\dot{\mathcal{F}}\right.$ is a $\mathrm{KH}\left(\aleph_{n}, \aleph_{0}\right)$-family $\urcorner$.

Let $\mathcal{F}=\dot{\mathcal{F}}[K]$, and let $A=\left\langle\mathcal{F} \uparrow X: X \in\left[\omega_{n}\right]^{\omega}\right\rangle$. Choose $\lambda<\kappa$ regular such that $A \in L\left[K_{\lambda}\right]$. Let $b \in \mathcal{F}$ be such that $b \notin L\left[K_{\lambda}\right]$.

From now on we work in $L\left[K_{\lambda}\right]$ and force with $\left(\mathbb{R}: \mathbb{R}_{\lambda}\right)$. Let $\dot{b}$ be an $\left(\mathbb{R}: \mathbb{R}_{\lambda}\right)$-name for $b$, and let $r_{0} \in\left(\mathbb{R}: \mathbb{R}_{\lambda}\right), r_{0}=\left\langle p_{0},\left\langle\left(X_{i, 0}, F_{i, 0}, g_{i, 0}\right): 0<i<n\right\rangle\right\rangle$, be such that

$$
r_{0} \|-\ulcorner\dot{b} \in \dot{\mathcal{F}} \text { and } \dot{b} \notin V\urcorner
$$

It is easy to prove the following.

Claim 2.10 For each $r \leq r_{0}, r=\left\langle p,\left\langle\left(X_{i}, F_{i}, g_{i}\right): 0<i<n\right\rangle\right\rangle$, there are two conditions $r_{1}=\left\langle p_{1},\left\langle\left(X_{i, 1}, F_{i, 1}, g_{i, 1}\right): 0<i<n\right\rangle\right\rangle, r_{2}=\left\langle p_{2},\left\langle\left(X_{i, 2}, F_{i, 2}, g_{i, 2}\right)\right.\right.$ : $0<i<n\rangle\rangle$ and some $\xi<\omega_{n}$ such that:

(a) $r_{1}, r_{2} \leq r$,

(b) $J_{i}\left(X_{i}\right) \in X_{i, m}$ for all $0<i<n$ and $m=1,2$,

(c) $\quad r_{1} \|-\ulcorner\check{\xi} \in \dot{b}\urcorner$ iff $r_{2} \|-\ulcorner\check{\xi} \notin \dot{b}\urcorner$.

Using the above, we can construct a sequence $\left\langle r_{s}=\left\langle p_{s},\left\langle\left(X_{i, s}, F_{i, s}, g_{i, s}\right): 0<\right.\right.\right.$ $\left.i<n\rangle\rangle: s \in^{<\omega} 2\right\rangle$ of conditions in $\left(\mathbb{R}: \mathbb{R}_{\lambda}\right)$ and a sequence $\left\langle\xi_{m}: m<\omega\right\rangle$ of elements of $\omega_{n}$ such that the following hold:

- $r_{s * m} \leq r_{s}$, for each $s \in^{<\omega} 2$ and $m<2$,

- $J_{i}\left(X_{i, s}\right) \in X_{i, s * m}$ for each $s \in^{<\omega} 2, m<2$ and $0<i<n$,

- $r_{s * 0} \|-\left\ulcorner\check{\xi}_{m} \in \dot{b}\right\urcorner$ iff $r_{s * 1} \|-\left\ulcorner\breve{\xi}_{m} \notin \dot{b}\right\urcorner$, where $m$ is the length of $s$.

Let $X=\left\{\xi_{m}: m<\omega\right\}$, and for each $f \in{ }^{\omega} 2$, using Claim 2.9, let $r_{f} \in\left(\mathbb{R}: \mathbb{R}_{\lambda}\right)$ be an extension of all of the $r_{f\lceil m}$ 's, $m<\omega$. For each $f$ as above, we can find some $q_{f} \leq r_{f}$ and some $b_{f} \in L\left[K_{\lambda}\right]$ such that

$$
q_{f} \|-\left\ulcorner\dot{b} \cap \check{X}=\check{b}_{f}\right\urcorner
$$

Note that $\mathcal{F} \uparrow X \supseteq\left\{b_{f}: f \in \in^{\omega} 2\right\}$ and for $f \neq g$ in ${ }^{\omega} 2$, we have $b_{f} \neq b_{g}$, and hence $\mathcal{F} \uparrow X$ must have size at least $2^{\aleph_{0}}$ which is in contradiction with our assumption.

It follows that $\mathrm{KH}\left(\aleph_{n}, \aleph_{0}\right)$ fails in $L[K]$. This completes the proof of Lemma 2.7. 


\section{Proof of Con $(b)$ implies Con $(a)$}

Now we show that if $n \geq 1$, and the Gap- $n$-Kurepa hypothesis fails, then there exists an inaccessible cardinal in $L$. In fact we will prove the following more general result.

Lemma 3.1 Suppose that $\lambda<\kappa$ are infinite cardinals such that $\kappa$ is regular, $\kappa^{\lambda}=\kappa$ and $\mathrm{KH}(\kappa, \lambda)$ fails. Then $\kappa^{+}$is an inaccessible cardinal in $L$.

The rest of this section is devoted to the prove of the above lemma. Assume on the contrary that the lemma fails. Thus we can find $X \subseteq \kappa$ such that:

- $V$ and $L[X]$ have the same cardinals up to $\kappa^{+}$,

- $\left([\kappa]^{\lambda}\right)^{V}=\left([\kappa]^{\lambda}\right)^{L[X]}$.

It follows that a $\mathrm{KH}(\kappa, \lambda)$-family in $L[X]$ is a real $\mathrm{KH}(\kappa, \lambda)$-family, and hence $\mathrm{KH}(\kappa, \lambda)$ fails in $L[X]$. The following lemma gives us the required contradiction.

Lemma 3.2 Suppose that $V=L[X]$, where $X \subseteq \kappa$. Then $\mathrm{KH}(\kappa, \lambda)$ holds.

Proof Our proof is very similar to the proof of Theorem 2 in [3]. We give it for completeness. For each $x \in[\kappa]^{\lambda}$ let

$$
M_{x}=\text { the smallest } M \prec L_{\kappa}[X] \text { such that } x \cup\{x\} \cup(\lambda+1) \subseteq M \text {. }
$$

Let $\mathcal{F}=\left\{t \subseteq \kappa: \forall x \in[\kappa]^{\lambda}, t \cap x \in M_{x}\right\}$. We show that $\mathcal{F}$ is a $\operatorname{KH}(\kappa, \lambda)$-family. It suffices to show that $\operatorname{Card}(\mathcal{F}) \geq \kappa^{+}$. Suppose not. Let $C=\left\langle t_{v}: v<\kappa\right\rangle$ be an enumeration of $\mathcal{F}$ definable in $L_{\kappa^{+}}[X]$. By recursion on $v<\kappa$, define a chain $\left\langle N_{v}: v<\kappa\right\rangle$ of elementary submodels of $L_{\kappa^{+}}[X]$ as follows:

$$
\begin{aligned}
& N_{0}=\text { the smallest } N \prec L_{\kappa^{+}}[X] \text { such that } \lambda \in N \text { and } N \cap \kappa \in \kappa, \\
& N_{\nu+1}=\text { the smallest } N \prec L_{\kappa^{+}}[X] \text { such that } N \cap \kappa \in \kappa \text { and } N_{\nu} \cup\left\{N_{\nu}\right\} \subseteq N, \\
& N_{\delta}=\bigcup_{\nu<\delta} N_{\nu} \text {, if } \delta \text { is a limit ordinal. }
\end{aligned}
$$

For each $v<\kappa$ set $\alpha_{v}=N_{v} \cap \kappa$. Using the condensation lemma for $L[X]$, we obtain an ordinal $\beta_{v}$ and an isomorphism $\sigma_{v}$ such that

$$
\sigma_{v}:\left\langle N_{\nu}, \in, N_{\nu} \cap X\right\rangle \simeq\left\langle L_{\beta_{v}}\left[X \cap \alpha_{\nu}\right], \in, X \cap \alpha_{\nu}\right\rangle
$$

Then:

- $\alpha_{v}<\beta_{v}<\alpha_{v+1}$,

- $\sigma_{v}(\kappa)=\alpha_{v}$,

- $\sigma_{v}(X)=X \cap \alpha_{v}$,

- $\sigma_{v}\left\lceil\alpha_{v}=i d \uparrow \alpha_{\nu}\right.$,

- $L_{\beta_{v}}\left[X \cap \alpha_{v}\right] \models\left\ulcorner\alpha_{v}\right.$ is a regular cardinal, and $\alpha_{v}$ is the largest cardinal $\urcorner$.

Let $t=\left\{\beta_{v}: \beta_{\nu} \notin t_{\nu}\right\}$. Clearly $t \neq t_{\nu}$ for all $v<\kappa$, and hence $t \notin \mathcal{F}$. Let $x \in[\kappa]^{\lambda}$ be such that: 
- $t \cap x \notin M_{x}$,

- $\alpha=\sup (x)$ is minimal.

It follows that $t \cap x$ is cofinal in $\alpha$, and hence $\alpha=\alpha_{\eta}$ for some $\eta<\kappa$. We have

$$
t \cap x=\left\{\beta_{v} \in x: \beta_{v}<\alpha_{\eta} \text { and } \beta_{v} \notin t_{v} \cap \alpha_{\eta}\right\}
$$

and thus $t \cap x$ is definable from $x,\left\langle\beta_{v}: v<\eta\right\rangle$ and $\left\langle t_{v} \cap \alpha_{\eta}: v<\eta\right\rangle$. It is clear that:

- $x \in M_{x}$

- $\left\langle\beta_{v}: v<\eta\right\rangle$ is definable in $L_{\beta_{\eta}}\left[X \cap \alpha_{\eta}\right]$.

- $\sigma_{\eta}(C)=\left\langle t_{v} \cap \alpha_{\eta}: v<\eta\right\rangle$, and hence $\left\langle t_{v} \cap \alpha_{\eta}: v<\eta\right\rangle$ is definable in $L_{\beta_{\eta}}\left[X \cap \alpha_{\eta}\right]$.

Clearly $X \cap \alpha_{\eta} \in M_{x}$. We show that $\beta_{\eta} \in M_{x}$. It will follow that $t \cap x \in M_{x}$ which is a contradiction. The proof is in a sequence of claims. Let $M=M_{x}$.

Claim 3.3 $\mathcal{P}\left(\alpha_{\eta}\right) \cap M \nsubseteq L_{\beta_{\eta}}\left[X \cap \alpha_{\eta}\right]$.

Proof Suppose not. Since $c f\left(\alpha_{\eta}\right)=c f(x) \leq \lambda<\alpha_{\eta}$, there is $a \in M$ such that $a \subseteq \alpha_{\eta}$ is cofinal in $\alpha_{\eta}$ and has order type less than $\alpha_{\eta}$. Then $a \in L_{\beta_{\eta}}\left[X \cap \alpha_{\eta}\right]$, and hence $\alpha_{\eta}$ is not a regular cardinal in $L_{\beta_{\eta}}\left[X \cap \alpha_{\eta}\right]$. A contradiction.

For $l<v<\kappa$ set:

- $\alpha^{(v)}=\left\langle\alpha_{\iota}: \iota \leq v\right\rangle$,

- $\beta^{(\nu)}=\left\langle\beta_{l}: \iota \leq \nu\right\rangle$

- $\sigma_{\iota v}=\sigma_{v} \sigma_{l}^{-1}:\left\langle L_{\beta_{l}}\left[X \cap \alpha_{l}\right], \in, X \cap \alpha_{\iota}\right\rangle \longrightarrow\left\langle L_{\beta_{v}}\left[X \cap \alpha_{v}\right], \in, X \cap \alpha_{\nu}\right\rangle$,

- $\sigma^{(v)}=\left\langle\sigma_{\iota v}: \iota<\tau \leq v\right\rangle$.

Claim 3.4 $v \in M \cap \eta$ implies $\alpha^{(v)}, \beta^{(v)}, \sigma^{(v)} \in M$.

Proof First note that $\alpha_{\nu} \in M$ implies $\alpha^{(v)} \in M$, since $\left\langle\alpha_{\iota}: \iota<v\right\rangle$ is definable from $L_{\beta_{v}}\left[X \cap \alpha_{\nu}\right]$ the way $\left\langle\alpha_{\iota}: \iota<\kappa\right\rangle$ was defined from $L_{\kappa^{+}}[X]$. It follows that $v \in M \cap \eta$ implies $\alpha^{(\nu)} \in M$, since there is $\tau, \nu \leq \tau<\eta$ such that $\alpha_{\tau} \in M$ and $\alpha_{\nu}=\alpha^{\tau}(\nu) \in M$. By similar arguments $v \in M \cap \eta$ implies $\beta^{(v)}, \sigma^{(v)} \in M$.

We note that

$$
\left\langle\left\langle L_{\beta_{l}}\left[X \cap \alpha_{l}\right], \in, X \cap \alpha_{\iota}\right\rangle_{l<\eta},\left\langle\sigma_{\iota v}\right\rangle_{l<v<\eta}\right\rangle
$$

is a directed system of elementary embeddings, and if

$$
\left\langle\langle U, E, Y\rangle,\left\langle g_{\iota}\right\rangle_{\iota<\eta}\right\rangle
$$

is its direct limit, then:

- $\langle U, E, Y\rangle \simeq\left\langle L_{\beta_{\eta}}\left[X \cap \alpha_{\eta}\right], \in, X \cap \alpha_{\eta}\right\rangle$,

- $g_{\iota}:\left\langle L_{\beta_{l}}\left[X \cap \alpha_{\iota}\right], \in, X \cap \alpha_{\iota}\right\rangle \longrightarrow\langle U, E, Y\rangle$,

- If $f:\langle U, E, Y\rangle \simeq\left\langle L_{\beta_{\eta}}\left[X \cap \alpha_{\eta}\right], \in, X \cap \alpha_{\eta}\right\rangle$, then $\sigma_{\iota \eta}=f g_{\iota}$. 
Now let $\pi:\langle M, \in, M \cap X\rangle \simeq\left\langle L_{\delta}[\tilde{X}], \in, \tilde{X}\right\rangle$, where $\tilde{X}=\pi[M \cap X]$. Let

- $\tilde{\alpha}^{(v)}=\pi\left(\alpha^{(v)}\right)$,

- $\tilde{\beta}^{(v)}=\pi\left(\beta^{(v)}\right)$,

- $\tilde{\sigma}^{(v)}=\pi\left(\sigma^{(v)}\right)$,

- $\tilde{\alpha}=\bigcup_{v \in M \cap \eta} \tilde{\alpha}^{(v)}$,

- $\tilde{\beta}=\bigcup_{v \in M \cap \eta} \tilde{\beta}^{(v)}$,

- $\tilde{\sigma}=\bigcup_{\nu \in M \cap \eta} \tilde{\sigma}^{(v)}$,

and

- $\tilde{\alpha}_{\iota}=\pi\left(\alpha_{\pi^{-1}(\iota)}\right)$,

- $\tilde{\beta}_{\iota}=\pi\left(\beta_{\pi^{-1}(\iota)}\right)$,

- $\tilde{\sigma}_{\iota v}=\pi\left(\sigma_{\pi^{-1}(\iota), \pi^{-1}(v)}\right)$.

Now

$$
\left\langle\left\langle L_{\tilde{\beta}_{l}}\left[\tilde{X} \cap \tilde{\alpha}_{\iota}\right], \in, \tilde{X} \cap \tilde{\alpha}_{\iota}\right\rangle_{l<\pi(\eta)},\left\langle\tilde{\sigma}_{\iota v}\right\rangle_{l<v<\pi(\eta)}\right\rangle
$$

is a directed system of elementary embeddings. Let

$$
\left\langle\langle\tilde{U}, \tilde{E}, \tilde{Y}\rangle,\left\langle\tilde{g}_{\iota}\right\rangle_{\iota<\pi(\eta)}\right\rangle
$$

be its direct limit. Then

- $\tilde{g}_{\iota}:\left\langle L_{\tilde{\beta}_{l}}\left[\tilde{X} \cap \tilde{\alpha}_{\iota}\right], \in, \tilde{X} \cap \tilde{\alpha}_{\iota}\right\rangle \longrightarrow\langle\tilde{U}, \tilde{E}, \tilde{Y}\rangle$,

- There is an elementary embedding $h$ such that the following diagram is commutative

$$
\begin{aligned}
&\left\langle L_{\beta_{\pi^{-1}(l)}}\left[X \cap \alpha_{\pi^{-1}(\iota)}\right], \in, X \cap \alpha_{\pi^{-1}(\iota)}\right) \stackrel{g_{\pi^{-1}(l)}}{\longrightarrow}\langle U, E, Y\rangle \\
& \pi^{-1} \uparrow \uparrow h \\
&\left\langle L_{\tilde{\beta}_{l}}\left[\tilde{X} \cap \tilde{\alpha}_{\iota}\right], \in, \tilde{X} \cap \tilde{\alpha}_{\iota}\right\rangle \stackrel{\tilde{g}_{\iota}}{\longrightarrow}\langle\tilde{U}, \tilde{E}, \tilde{Y}\rangle
\end{aligned}
$$

It follows that $\langle\tilde{U}, \tilde{E}\rangle$ is well founded. Let

$$
\tilde{f}:\langle\tilde{U}, \tilde{E}, \tilde{Y}\rangle \simeq\left\langle L_{\bar{\beta}}[\bar{X}], \in, \bar{X}\right\rangle .
$$

Also let

- $\bar{\sigma}_{\iota}=\tilde{f} \tilde{g}_{\iota}:\left\langle L_{\tilde{\beta}_{l}}\left[\tilde{X} \cap \tilde{\alpha}_{\iota}\right], \in, \tilde{X} \cap \tilde{\alpha}_{\iota}\right\rangle \longrightarrow\left\langle L_{\bar{\beta}}[\bar{X}], \in, \bar{X}\right\rangle$,

- $\pi^{*}=f h \tilde{f}^{-1}:\left\langle L_{\bar{\beta}}[\bar{X}], \in, \bar{X}\right\rangle \longrightarrow\left\langle L_{\beta_{\eta}}\left[X \cap \alpha_{\eta}\right], \in, X \cap \alpha_{\eta}\right\rangle$. 
Then $\tilde{\sigma}_{\iota \tau}=\bar{\sigma}_{\tau}^{-1} \bar{\sigma}_{\iota}$ for $\iota<\tau<\pi(\eta)$, and the following diagram is commutative

$$
\begin{aligned}
&\left\langle L_{\beta_{\pi^{-1}(l)}}\left[X \cap \alpha_{\pi^{-1}(\iota)}\right], \in, X \cap \alpha_{\pi^{-1}(\iota)}\right\rangle \stackrel{\sigma_{\pi^{-1}(l), \eta}}{\longrightarrow}\left\langle L_{\beta_{\eta}}\left[X \cap \alpha_{\eta}\right], \in, X \cap \alpha_{\eta}\right\rangle \\
& \pi^{-1} \uparrow \uparrow \pi^{*} \\
&\left\langle L_{\tilde{\beta}_{l}}\left[\tilde{X} \cap \tilde{\alpha}_{\iota}\right], \in, \tilde{X} \cap \tilde{\alpha}_{\iota}\right\rangle \stackrel{\bar{\sigma}_{\iota}}{\longrightarrow}\left\langle L_{\bar{\beta}}[\bar{X}], \in, \bar{X}\right\rangle
\end{aligned}
$$

Let $\bar{\alpha}$ be such that $L_{\bar{\beta}}[\bar{X}] \models\ulcorner\bar{\alpha}$ is the largest cardinal $\urcorner$.

Claim 3.5 (a) $\pi\left(\alpha_{\eta}\right)=\bar{\alpha}$,

(b) $\pi^{*}(\bar{\alpha})=\alpha_{\eta}$,

(c) $\pi^{*} \uparrow \bar{\alpha}=i d \uparrow \bar{\alpha}$.

Proof (a) Follows easily from the facts that $\bar{\alpha}=\sup _{\iota<\eta} \tilde{\alpha}_{\iota}, \alpha_{\eta}=\sup _{\iota \in M \cap \eta} \alpha_{\iota}$ and $\pi^{-1}\left(\tilde{\alpha}_{\iota}\right)=\alpha_{\pi^{-1}(\iota)}$. (b) Follows from the choice of $\bar{\alpha}$ and the elementarily of $\pi^{*}$. (c) Is trivial, as $\bar{\alpha} \subseteq L_{\bar{\beta}}[\bar{X}]$.

Next we have

Claim 3.6 If $a \subseteq \bar{\alpha}$ and $a \in L_{\bar{\beta}}[\bar{X}] \cap L_{\delta}[\tilde{X}]$, then $\pi^{*}(a)=\pi^{-1}(a)$.

Proof Since $a \subseteq \bar{\alpha}, \pi^{*}(a), \pi^{-1}(a) \subseteq \alpha_{\eta}$, and hence $\pi^{*}(a)=\bigcup_{v \in M \cap \eta} \pi^{*}(a) \cap v=$ $\bigcup_{v<\pi(\eta)} \pi^{*}(a \cap v) \stackrel{\text { claim } 3.5}{=} \bigcup_{\nu<\pi(\eta)} \pi^{-1}(a \cap v)=\pi^{-1}(a)$.

Claim $3.7 \delta>\bar{\beta}$.

Proof Suppose not. Then $\delta \leq \bar{\beta}$ and $\pi^{*} \pi$ maps $M$ into $L_{\beta_{\eta}}\left[X \cap \alpha_{\eta}\right]$, and by claim 3.6, $\pi^{*} \pi(a)=a$ for $a \subseteq \alpha_{\eta}, a \in M$. It follows that $\mathcal{P}\left(\alpha_{\eta}\right) \cap M \subseteq L_{\beta_{\eta}}\left[X \cap \alpha_{\eta}\right]$, which is in contradiction with claim 3.3.

It follows that $\bar{\beta} \in L_{\delta}[\tilde{X}]$ and hence $\tilde{\beta}=\left\langle\tilde{\beta}_{\iota}: \iota<\pi(\eta)\right\rangle \in L_{\delta}[\tilde{X}]$, since $\tilde{\beta}$ is definable from $L_{\bar{\beta}}[\bar{X}]$ as $\left\langle\tilde{\beta}_{\iota}: \iota<\kappa\right\rangle$ was defined from $L_{\kappa^{+}}[X]$. Similarly $\tilde{\sigma}=\left\langle\tilde{\sigma}_{\iota, v}\right.$ : $\iota<v<\pi(\eta)\rangle \in L_{\delta}[\tilde{X}]$. It is easily seen that

Claim 3.8 (a) $\pi^{-1}(\tilde{\alpha})=\left\langle\alpha_{\iota}: \iota<\eta\right\rangle$,

(b) $\pi^{-1}(\tilde{\beta})=\left\langle\beta_{\iota}: \iota<\eta\right\rangle$,

(c) $\pi^{-1}(\tilde{\sigma})=\left\langle\sigma_{\iota v}: \iota<v<\eta\right\rangle$.

Now note that:

- $L_{\bar{\beta}}[\bar{X}]$ is the direct limit of $L_{\tilde{\beta}_{\iota}}\left[\tilde{X} \cap \tilde{\alpha}_{\iota}\right], \tilde{\sigma}_{\iota v}, \iota<v<\pi(\eta)$,

- $\pi^{-1}[\bar{X}]=X \cap \alpha_{\eta}$,

- $\pi^{-1}\left[\tilde{X} \cap \tilde{\alpha}_{\iota}\right]=X \cap \alpha_{\iota}$,

and hence by elementarily of $\pi^{-1}, L_{\pi^{-1}(\bar{\beta})}\left[X \cap \alpha_{\eta}\right]$ is the direct limit of $L_{\beta_{l}}\left[X \cap \alpha_{\iota}\right]$, $\sigma_{\iota v}, \iota<v<\eta$.

It follows that $\pi^{-1}(\bar{\beta})=\beta_{\eta} \in M$. We are done. 


\section{Open problems}

We close the paper with some remarks and open problems.

By the results of Vaught, Chang, Jensen (see [1], Chapter VIII) and Silver (see [7]), it is consistent, relative to the existence of an inaccessible cardinal, to have the Gap- $n$-transfer principle with the failure of the gap- $(n+1)$-transfer principle for $n=1$. The answer is unknown for $n>1$.

Question 4.1 Let $n>1$. Is it consistent to have the Gap-n-transfer principle with the failure of the Gap- $(n+1)$-transfer principle?

Another related question is

Question 4.2 Let $n>1$. Is it consistent to have $(\kappa, n)$-morasses for each uncountable regular $\kappa$, but no $\left(\omega_{1}, n+1\right)$-morasses?

Remark 4.3 Assuming the existence of large cardinals, it is possible to build a model of set theory in which there exists a $(\kappa, 1)$-morass for each uncountable regular $\kappa$, but there are no $\left(\omega_{1}, 2\right)$-morasses.

In the literature the canonical counter-example to the Gap-1-transfer principle is the non-existence of Special Aronszajn trees (see [5]). T. Raesch, in his dissertation (see [6]), showed that this principle can fail in the presence of such trees. On the other hand the canonical counter-example to the Gap-2-transfer principle is the non-existence of Kurepa trees (see [7]). Inspired by the work of Raesch, Jensen produced, relative to the existence of a Mahlo cardinal, a model in which the Gap-2-transfer principle fails, while the Gap-1-Kurepa hypothesis holds (see [4]). However the following is open.

Question 4.4 Is it consistent relative to an inaccessible cardinal to have the Gap1-Kurepa Hypothesis but a failure of the Gap-2-transfer principle?

Remark 4.5 It is possible to show that the existence of an $\left(\omega_{2}, 1\right)$-morasses implies $\mathrm{KH}\left(\aleph_{2},<\aleph_{2}\right)$. Thus in our model, for $n=2$, the Gap-1-Kurepa hypothesis holds, while in it there are no $\left(\omega_{2}, 1\right)$-morasses.

Question 4.6 Let $n>1$. Is it consistent with $G C H$ to have $\operatorname{KH}\left(\aleph_{n}, \aleph_{0}\right)$ but not $\mathrm{KH}\left(\aleph_{n}, \aleph_{1}\right)$ ?

Question 4.7 Let $n>1$. Is it consistent with $G C H$ to have $\mathrm{KH}\left(\aleph_{n}, \aleph_{i}\right)$ for all $i<n$, but not $\mathrm{KH}\left(\aleph_{n},<\aleph_{n}\right)$ ?

Acknowledgments This work was done when the second author was at the Kurt Gödel Research Center. He would like to thank Prof. Friedman for his inspiration and encouragement. The authors wish to thank the Austrian Research Fund (FWF) for its generous support through Project P 21968-N13.

\section{References}

1. Devlin, K.J.: Constructibility, Perspectives in Mathematical Logic. Springer-Verlag, Berlin (1984)

2. Jech, T.: Set Theory. Academic Press, New York (1978) 
3. Jensen, R.: Some combinatorial properties of $L$ and $V$. http://www.mathematik.huberlin.de/raesch/org/ jensen.html

4. Jensen, R.: Remarks on the two cardinal problem. http://www.mathematik.hu-berlin.de/raesch/org/ jensen.html

5. Mitchell, W.: Aronszajn trees and the independence of the transfer property. Ann. Math. Log. 5, 21-46 (1972)

6. Raesch T.: On the failure of the GAP-1 transfer property, Ph.D. thesis. http://www.math.uni-bonn.de/ people/raesch/publicationen.html (2005)

7. Silver, J: The independence of Kurepa's conjecture and two cardinal conjectures in model theory. In: Scott, D. (ed.) "Axiomatic set theory," proc. symp. Pure Math. vol. 13, 1, pp. 383-390. Am. Math. Soc., Providence Rhode Island, (1971) 